\title{
Erratum to: Microstructural and biomechanical alterations of the human aorta as a function of age and location
}

\author{
Darren Haskett • Gregory Johnson • Aifang Zhou • \\ Urs Utzinger · Jonathan Vande Geest
}

Published online: 12 July 2012

(C) Springer-Verlag 2012

Erratum to: Biomech Model Mechanobiol (2010) 9:725736 DOI 10.1007/s10237-010-0209-7

Unfortunately, the units given in columns 2 and 3 of Table 4 were mislabeled. Table 4 should be correctly shown as:

The online version of the original article can be found under doi:10.1007/s10237-010-0209-7.

D. Haskett · U. Utzinger · J. Vande Geest

Graduate Interdisciplinary Program of Biomedical Engineering,

The University of Arizona, 1657 E. Helen St.,

PO Box 210240, Tucson, AZ 85721-0240, USA

G. Johnson

Agriculture and Biosystems Engineering,

The University of Arizona, 1177 E 4th St. Room 403,

PO Box 210038, Tucson, AZ 85721-0038, USA
A. Zhou $\cdot$ J. Vande Geest $(\varangle)$
Department of Aerospace and Mechanical Engineering,
The University of Arizona, 1130 N. Mountain,
PO Box 210119, Tucson, AZ 85721-0119, USA
e-mail: jpv1@email.arizona.edu
U. Utzinger - J. Vande Geest
BIO5 Institute, The University of Arizona, 1657 E. Helen St.,
PO Box 210240, Tucson, AZ 85721-0240, USA
U. Utzinger · J. Vande Geest
Department of Biomedical Engineering, The University of Arizona,
1657 E. Helen St., PO Box 210240, Tucson, AZ 85721-0240, USA 
Table 4 Material parameter means determined from the Holzapfel model given for each location and age group

\begin{tabular}{|c|c|c|c|c|c|c|}
\hline $\begin{array}{l}\text { Holzapfel model } \\
\text { Location }\end{array}$ & $C_{10}(\mathrm{kPa})$ & $k_{1}(\mathrm{MPa})$ & $k_{2}$ & $\kappa$ & $\theta\left(^{\circ}\right)$ & $K(\mathrm{GPa})$ \\
\hline Ascending $1(0-30)$ & 20.9 & 0.71 & 0.40 & 0.08 & 44.4 & 20.5 \\
\hline Ascending 2 (31-60) & 16.6 & 0.79 & 3.13 & 0.13 & 44.1 & 24.9 \\
\hline Ascending 3 (61-above) & 44.6 & 2.07 & 93.33 & 0.18 & 45.5 & 36.4 \\
\hline Arch $1(0-30)$ & 30.8 & 0.93 & 4.64 & 0.14 & 46.1 & 37.0 \\
\hline Arch $2(31-60)$ & 22.0 & 1.02 & 5.89 & 0.17 & 44.7 & 37.7 \\
\hline Arch 3 (61-above) & 65.5 & 2.53 & 134.59 & 0.17 & 48.2 & 38.7 \\
\hline Descending $1(0-30)$ & 29.7 & 1.34 & 1.79 & 0.10 & 44.5 & 29.5 \\
\hline Descending 2 (31-60) & 35.1 & 1.93 & 39.64 & 0.20 & 44.3 & 32.9 \\
\hline Descending 3 (61-above) & 63.3 & 3.22 & 145.35 & 0.20 & 51.4 & 55.2 \\
\hline Suprarenal 1 (0-30) & 28.8 & 0.86 & 2.83 & 0.10 & 45.3 & 30.4 \\
\hline Suprarenal 2 (31-60) & 32.3 & 1.35 & 22.45 & 0.17 & 45.9 & 41.3 \\
\hline Suprarenal 3 (61-above) & 55.4 & 2.77 & 187.34 & 0.15 & 48.7 & 60.6 \\
\hline Abdominal $1(0-30)$ & 50.9 & 2.37 & 107.13 & 0.16 & 46.7 & 48.1 \\
\hline Abdominal 2 (31-60) & 37.7 & 1.78 & 114.69 & 0.18 & 46.6 & 29.0 \\
\hline Abdominal 3 (61-above) & 100.9 & 4.07 & 165.55 & 0.16 & 48.4 & 75.5 \\
\hline
\end{tabular}

